\title{
A LOW-COST ARCHITECTURE OF AUDIOMETER BASED ON DIRECT DIGITAL SYNTHESIZER
}

\author{
Han-Chang $\mathrm{Wu}^{1}$, Student Member, Li-Ping Yang ${ }^{1}$, Chien-Hao Chen ${ }^{1}$, \\ Shuenn-Tsong Young ${ }^{2}$, and Te-Son Kuo ${ }^{1,3}$, Senior Member \\ ${ }^{1}$ Department of Electrical Engineering, National Taiwan University \\ ${ }^{2}$ Institute of Biomedical Engineering, National Yang-Ming University \\ ${ }^{3}$ Graduate Institute for Biomedical Engineering, National Taiwan University \\ E-mail:wuman@tpts1.seed.net.tw
}

\begin{abstract}
In this paper we purposed a new architecture of audiometer. By utilizing the direct digital synthesizer (DDS), pure tone testing signals could be generated more precisely than other existing techniques. A microcontroller was used to receive the stimulating commands from PC and then send appropriate control signals to the DDS and the $\log$ attenuator. All components were assembled with compact size and low cost.
\end{abstract}

Keywords: audiometer, direct digital synthesizer.

\section{Introduction}

Audiometers are utilized as a basic testing apparatus for human hearing. Two testing methods, pure-tone stimulating and speech hearing with/without noise masking, are used. Pure-tone testing is more fundamental, and is intended for the measurement of sensitivity and hearing threshold level of the required frequency. Pure-tone stimulating signals can be generated by either analog or digital circuits. Digital circuits that are much well for frequency precision are adapted by more and more audiometers [1-3].

\section{Methods}

The system block diagram is illustrated in figure 1. A singlechip of direct digital synthesizer (DDS) (AD9850, Analog Devices) was utilized to generator pure sinusoidal signals. Its frequency was controlled by a microcontroller (AT89C52, ATMEL). The microcontroller received the stimulating parameters from PCs via RS-232, including frequency, amplitude, and stimulating modes. At each specified stimulating periods, the microcontroller changed the frequency and amplitude if necessary by sending controlling signals to DDS and a log attenuator (DS1807, DALLAS).

\section{Results}

The architecture could achieve the following specifications that satisfied the general requirements of the audiometer.

\begin{tabular}{ll}
\hline Frequency Range & $1 \sim 20000 \mathrm{~Hz}$ \\
\hline Signal Level & $0 \sim 90 \mathrm{~dB}$ (Full Range) \\
\hline SNR & $>70 \mathrm{~dB}$ \\
\hline
\end{tabular}

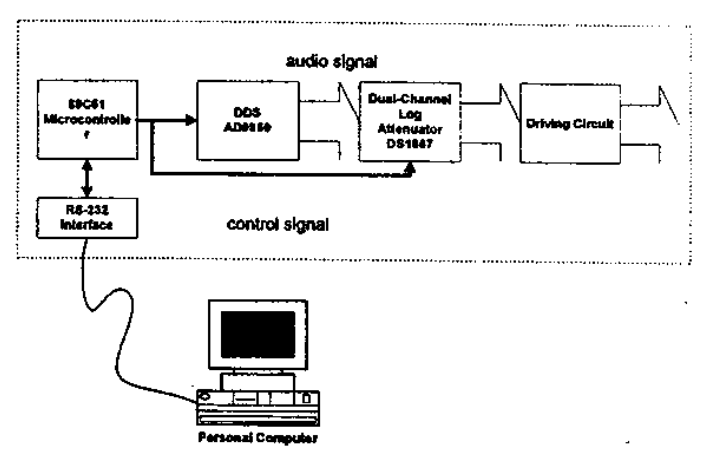

Figure1. System block diagram

4. Discussion

To generate high precision and flexible pure-tone signal, digital signal processor (DSP) must be used and thus the system would be more expensive and complex. In the study, we propose a new architecture of the audiometer based on a direct digital synthesizer (DDS). DDS is a novel technique to generate high precision sinusoidal signal and is originally used in communication applications [4]. In our system, we used a single-chip DDS as the pure-tone generator to implement a low-cost, high precision audiometer.

\section{Reference}

1. Stewart, R.W et al., "A digital signal processing audiometric workstation," Audio DSP - Circuits and Systems, IEE Colloquium on, pp. 7/1 -7/5, 1993

2. Mudduveerappa et al., "Microprocessor based audiometer for mass screening," Proceedings of the Annual International Conference of the IEEE, pp. 1625, vol.4, 1988.

3. Martinez-Baez, C.A. et al., "Manual And Automatic Programmable Audiometer," Proceedings of the Annual International Conference of the IEEE, pp. 1915-1916, vol. 13, 1991.

4. S. Brigati et al., "Digital Synthesis of Analog signals for Audiometric Applications, " IEEE International Symposium on Circuit and Systems, 1997 